\section{Association of adult mastocytosis with M541L in the transmembrane domain of KIT}

\section{Editor}

Mastocytosis is a disorder characterized by mast cell (MC) proliferation and accumulation within various organs, most commonly the skin. Multiple KIT mutations have been reported in patients with adult mastocytosis, but the potential association of specific KIT mutations with specific subtypes of mastocytosis remains to be clarified.

We present the case of a 32-year-old caucasian woman who came to the Dermatology Department complaining of a 12-year history of multiple, generalized and reddish-brown macules and papules with positive Darier's sign. These lesions were more numerous in the trunk and limbs, and absent from face, palms and soles.

She had no systemic symptoms and physical examination was unremarkable.

Skin biopsy showed MC infiltrates in the dermis (Fig. 1), confirming the diagnosis of cutaneous mastocytosis.

Full blood count, white blood cell differential and routine serum biochemistry tests were within normal range. Serum tryptase level was $17.9 \mathrm{ng} / \mathrm{mL}$ (normal: $0.1-11.4 \mathrm{ng} / \mathrm{mL}$ ).

The patient had a 46,XX karyotype.

Abdominal ultrasound, PET scan and bone densitometry were all normal.

Complete bone marrow study showed normal percentage of bone marrow MCs (BMMCs) (0.04\%) which showed aberrant coexpression of CD25+, CD2+ and also CD33+, CD35+, CD45+, CD63+, CD69+ and CD59+. Analysis of coding exons 8, 9, 10, 11 and 17 of the KIT gene revealed the presence of a heterozygous substitution in exon 10, resulting in the substitution of leucine for methionine at codon 541 (M541L) (Fig. 2). The sequencing of

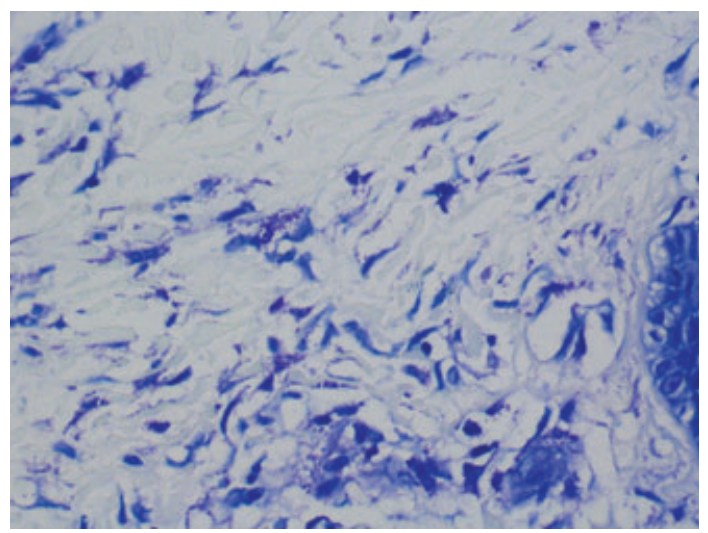

Figure 1 Mast cell infiltrates in the dermis; toluidine blue stain, $\times 400$.

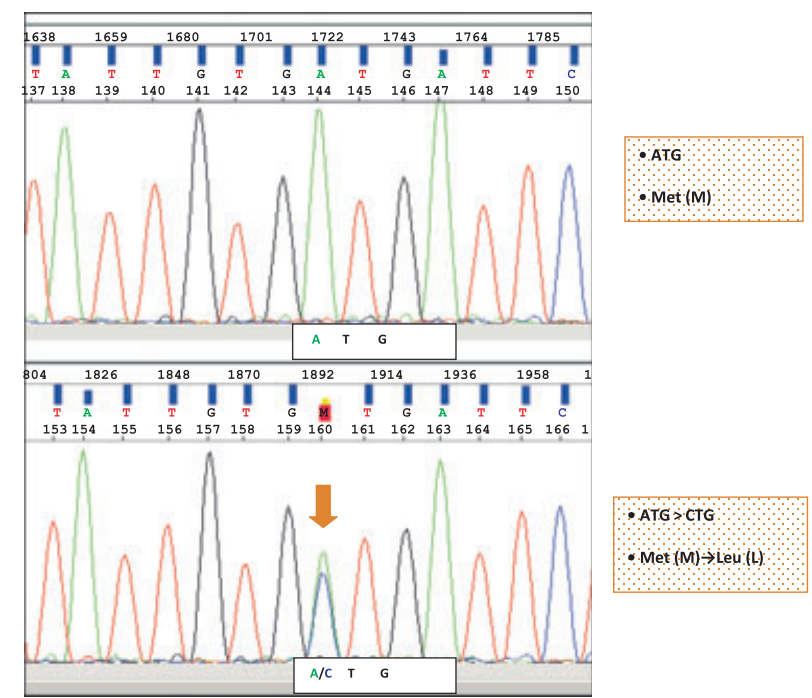

Figure 2 Analysis of coding exons 8, 9, 10, 11 and 17 of the KIT gene revealed the presence of a heterozygous substitution in exon 10, resulting in the substitution of leucine for methionine at codon 541 (M541L).

KIT exon 10, using DNA extracted from oral mucosa cells, confirmed that this was a germline mutation.

Human KIT is a protooncogene located at chromosome $4 \mathrm{q} 12$ that contains 21 exons, which encode a transmembrane receptor (kit) with tyrosine kinase (TK) activity. Binding of kit ligand stem cell factor (SCF) - to kit receptor is known to activate kit TK, which results in different biological effects depending on the activated cell. In MCs, these effects include, among others, cell proliferation and suppression of apoptosis. ${ }^{1}$

Mutations of KIT are considered to play a key role in the pathogenesis of mastocytosis. Most frequently, KIT mutations occur at exon 11 and 17, resulting in aminoacid changes at the juxtamembrane and TK domain of kit respectively. ${ }^{2}$ Furitsu et al. showed that point mutations in KIT (Asp816Val and Val560Asp) are capable of inducing constitutive activation of kit. ${ }^{3}$ Multiple other activating KIT mutations have been reported in patients with adult mastocytosis, but few are described in the transmembrane domain of KIT, ${ }^{1}$ encoded by exon 10. In our patient, we detected a heterozygous substitution (germline mutation) in exon 10, resulting in the substitution of leucine for methionine at codon 541 (M541L). This mutation was described in general population, ${ }^{4}$ in a family with piebaldism ${ }^{5}$ and in two unrelated pairs of apparently identical twin children with mastocytosis. ${ }^{6}$ To the best of our knowledge, however, this is the first time it is reported in an adult patient with mastocytosis. According to Foster et al., cells expressing M541L displayed a significantly heightened response to low levels of SCF, ${ }^{6}$ suggesting that these cells may have a proliferative and/or survival advantage.

Although our patient had elevated serum tryptase levels, coexpression of both $\mathrm{CD} 2$ and CD25 by BMMCs and a KIT 
mutation, she did not meet the World Health Organization criteria for systemic mastocytosis. ${ }^{7}$ However, it is important to underline the presence of the aberrant CD25 and CD2 immunophenotype, as this is present in virtually all patients with systemic mastocytosis. $^{2}$ Additionally, CD25 seems to be a marker for neoplastic MCs and its expression indicates histologically occult bone marrow infiltration, ${ }^{8}$ which could be an indication for additional investigation. ${ }^{9}$

In conclusion, we would like to emphasize that this is the first time that M541L KIT mutation is reported in an adult patient with mastocytosis. Although the role of this mutant form of KIT in the pathogenesis of the disease is still unclear, in our patient, it is associated with aberrant MC immunophenotype. From our point of view, although without systemic mastocytosis criteria, these patients should maintain regular clinical evaluation. Further studies will be required as all these are important prognosis factors determining these patients' follow-up.

\section{J Rocha, ${ }^{\dagger, \star}$ M Luz Duarte, ${ }^{\dagger}$ H Marques, ${ }^{\ddagger}$ F Torres, ${ }^{\S}$ P Tavares, ${ }^{\S}$ A Silva, ${ }^{\circledR}$ C Brito $^{\dagger}$ \\ Departments of ${ }^{\dagger}$ Dermatology and ${ }^{\ddagger}$ Oncology, Hospital de Braga, Braga, ${ }^{\S}$ Centro de Genética Clínica, Porto, and "Department of Pathology, Hospital de Braga, Braga, Portugal \\ *Correspondence: J Rocha. E-mail: joanacgomesrocha@hotmail.com}

\section{References}

1 Orfao A, Garcia-Montero AC, Sanchez L, Escribano L. Recent advances in the understanding of mastocytosis: the role of KIT mutations. $\mathrm{Br} \mathrm{J}$ Haematol 2007; 138: 12-30.

2 Garcia-Montero AC, Jara-Acevedo M, Teodosio C et al. KIT mutation in mast cells and other bone marrow hematopoietic cell lineages in systemic mast cell disorders: a prospective study of the Spanish Network on Mastocytosis (REMA) in a series of 113 patients. Blood 2006; 108: 2366-2372.

3 Furitsu T, Tsujimura T, Tono $\mathrm{T}$ et al. Identification of mutations in the coding sequence of the proto-oncogene c-kit in a human mast cell leukemia cell line causing ligand-independent activation of c-kit product. J Clin Invest 1993; 92: 1736-1744.

4 Krüger S, Emig M, Lohse P et al. The c-kit (CD117) sequence variation M541L, but not N564K, is frequent in the general population, and is not associated with CML in Caucasians. Leukemia 2006; 20: 354-355.

5 Murakami T, Fukai K, Oiso N et al. New KIT mutations in patients with piebaldism. J Dermatol Sci 2004; 35: 29-33.

6 Foster R, Byrnes E, Meldrum C et al. Association of paediatric mastocytosis with a polymorphism resulting in an amino acid substitution (M541L) in the transmembrane domain of c-KIT. Br J Dermatol 2008; 159: 1160-1169.

7 Valent P, Horny HP, Escribano L et al. Diagnostic criteria and classification of mastocytosis: a consensus proposal. Leuk Res 2001; 25: 603-625.

8 Pardanani A, Kimlinger T, Reeder T et al. Bone marrow mast cell immunophenotyping in adults with mast cell disease: a prospective study of 33 patients. Leuk Res 2004; 28: 777-783.

9 Hollmann TJ, Brenn T, Hornick JL. CD25 expression on cutaneous mast cells from adult patients presenting with urticaria pigmentosa is predictive of systemic mastocytosis. Am J Surg Pathol 2008; 32: 139-145.

DOI: $10.1111 / \mathrm{j} .1468-3083.2010 .03599 . x$

\section{Achilles' heel in dermatology}

\section{Editor}

We read with interest the case report of intertriginous drug eruption (baboon syndrome) induced by loflazepate ethyl, described by Watanabe et al. ${ }^{1}$ In particular, we appreciated how the authors solved the diagnostic doubt between friction dermatitis and drug eruption with the comprehensive diagnosis of drug eruption restrictedly localized on the frictional sites.

Dermatological literature is replete with similar cases where one or more cutaneous districts, for different reasons, become prone to harbouring heterogeneous skin disorders. For example, in 2002, Carrasco et al. published a case of drug eruption secondary to acyclovir with recall phenomenon in a dermatome previously affected by herpes zoster. ${ }^{2}$ The eruption, consisting of small erythematous macules and papules, was widespread, but the most striking feature was represented by the confluence of the lesions along the dermatome previously involved by herpes zoster. The authors considered it as an example of recall dermatitis (or recall phenomenon) since the generalized rash due to acyclovir was more intense in the dermatome previously compromised by the herpetic infection. Recall phenomenon, however, refers to drug-induced (often chemotherapy-induced) reactivation of an antecedent tissue damage caused by radiotherapy, sunburn, mechanical injury or allergen injections. In the case reported by Carrasco et al., no irradiation, no trauma, no immunization had previously 'marked' the cutaneous region that became the site of the lesional confluence. The only damage suffered by this region had been the herpetic infection, which itself could have compromised the local immune balance thus favouring a particular concentration of the acyclovir-induced rash. In this light, the above case report could be viewed as an example of Wolf's isotopic response, which refers to the occurrence of a new skin disorder (in this case a drug-reaction) at the site of a previous, unrelated and healed cutaneous disease, in most cases as a herpes zoster infection. ${ }^{3,4}$ The question was already raised by Mizukawa and Shiohara, who also took into consideration the possibility of a fixed drug eruption appearing in a striking linear pattern as a consequence of the previous zoster episode. ${ }^{5}$

We think that this question and similar ones are merely semantic questions. In fact, recall dermatitis, Wolf's isotopic response and fixed-drug eruption can all be considered as different aspects of a novel, unifying concept, named the immunocompromised district, ${ }^{6}$ which somehow evokes the ancient, mythological notion of Achilles' heel. The concept encompasses heterogeneous clinical events, such as herpetic infections, radiation dermatitis, sunburns, mechanical (including frictional) traumas, vaccinations and chronic lymph stasis, which can selectively damage and immunologically mark the cutaneous region they act upon. After the causing event has disappeared, the affected region may show clinically normal, but its immune behaviour often remains compromised forever. The immunocompromised district becomes a weak, 